\section{Revisión sistemática de la literatura sobre telemedicina}

La telemedicina consiste en el uso de las tecnologías de la información y de las comunicaciones para proporcionar servicios de atención sanitaria a personas que se encuentran lejos de los profesionales sanitarios. Se supone que la telemedicina pueda mejorar la asistencia y la equidad de la distribución de los servicios al incrementar la accesibilidad, especialmente en zonas remotas. No obstante, como ocurre con cualquier técnica nueva, antes de adoptar un uso generalizado de la telemedicina, es necesario demostrar su superioridad con respecto al método que pretende sustituir.

En este estudio, los autores analizan las pruebas existentes acerca de la eficacia y eficiencia económica de la telemedicina, con el fin de proporcionar bases sólidas para la toma de decisiones relacionadas con la implantación de estos servicios.

Se efectuó una búsqueda bibliográfica en varias bases de datos para encontrar estudios acerca de la telemedicina. Los criterios de inclusión de los estudios fueron los siguientes: que consideraran de forma científicamente válida los resultados de alguna forma de telemedicina en relación con los cambios administrativos, el desenlace de los pacientes o la evaluación económica, y que compararan la aplicación de la telemedicina con una alternativa convencional. Se excluyeron los estudios no controlados y los que solo describieran la factibilidad de la telemedicina o solo evaluaran técnicamente algún sistema. También se excluyeron los artículos duplicados. La calidad metodológica de los estudios que no consistieran únicamente en análisis económicos se clasificó, por orden decreciente, del siguiente modo: 1) metaanálisis de ensayos clínicos aleatorizados controlados; 2) ensayos clínicos aleatorizados controlados con muestras amplias; 3) ensayos clínicos aleatorizados controlados con muestras reducidas; 4 ) estudios prospectivos controlados no aleatorizados; 5) estudios retrospectivos controlados no aleatorizados; 6) estudios de cohortes; 7) estudios de casos y controles; 8) series clínicas no controladas, estudios descriptivos y métodos de consenso, y 9) informes de casos o informes anecdóticos.

Se identificaron 1124 artículos, entre los cuales se seleccionaron 133 para un examen más detallado. Se consideró que 50 cumplían los criterios de inclusión: 14 analizaron la aplicación de la telemedi- cina a diferentes tipos de consultas médicas; 7 a la monitorización o asesoramiento de los pacientes; 13 a la telerradiología, y los demás a los servicios de urgencias, psiquiatría, dermatología, cardiología, oftalmología o anatomía patológica. Dieciséis estudios consistieron fundamentalmente en análisis económicos, mientras que los otros 34 evaluaron algún parámetro clínico. En lo que se refiere a la calidad metodológica de estos 34 estudios, seis se incluyeron en el nivel 2-3, cuatro en el nivel 4-5, trece en el nivel 6, seis en el nivel 7 y cinco en el 8. En $30(60 \%)$ estudios se incluyó algún tipo de análisis económico.

La mayoría de los análisis económicos consistieron en variantes de análisis de costos. En tres estudios se afirmaba haberse realizado análisis de costo-beneficio, pero, desde el punto de vista metodológico, eran, más que nada, estudios de análisis de costos, una vez que los beneficios se estimaron como ahorros (sobre todo de costos de viaje) en comparación con la alternativa convencional. Tres de los cuatro estudios sobre la transmisión de imágenes diagnósticas indicaron que la telemedicina era más cara que la alternativa más barata. Los análisis económicos revelaron que la telerradiología, en particular la transmisión de imágenes de tomografía computadorizada, puede ahorrar costos, aunque uno de estos estudios, con buena calidad metodológica, no confirmó este dato. En general, la calidad de los análisis económicos fue baja.

Esta revisión demuestra que todavía existen pocos datos sobre la eficacia y la relación costoefectividad de la telemedicina. De los más de 1000 artículos revisados, la gran mayoría eran informes sobre la factibilidad de diferentes aplicaciones y fueron muy pocos los que incluyeron comparaciones con métodos convencionales alternativos. Por el momento, las pruebas más convincentes de la eficacia de la telemedicina están relacionadas con su aplicación en la telerradiología, la teleneurocirugía (transmisión de imágenes de tomografía computadorizada antes de la transferencia del paciente), la telepsiquiatría, la transmisión de datos electrocardiográficos y el uso de consultas electrónicas (correo electrónico y videoconferencia) entre los profesionales. No obstante, incluso para estas aplicaciones, la mayor parte de la literatura existente trata de estudios piloto y de resultados a corto plazo y en muchos casos se analizó la eficacia de la aplicación, más que su efectividad. Los estudios sobre la relación costo-efectividad de la telemedicina siguen siendo 
escasos. (Roine R, Ohinmaa A, Hailey D. Assessing telemedicine: a systematic review of the literature. CMAJ 2001;165:765-771.)

\section{El tratamiento del dolor de garganta con antibióticos en atención primaria}

El dolor de garganta es la segunda causa más frecuente de consulta, tras la tos. La mayoría de los casos son debidos a infecciones víricas. La principal causa bacteriana son los estreptococos $\beta$-hemolíticos del grupo A (EBA), que se aíslan en el 15 a $36 \%$ de los niños y el 5 a $17 \%$ de los adultos con dolor de garganta. Aunque esta es la única causa frecuente que requiere tratamiento con antibióticos, en 1992 la proporción de pacientes diagnosticados de faringitis que recibieron antibióticos fue muy superior $(76 \%$ de los adultos y $71 \%$ de los niños). En estos casos, los antibióticos recomendados como tratamiento de primera línea son la penicilina o la eritromicina, esta última en caso de alergia a la penicilina.

En este estudio, los autores utilizaron los datos de la Encuesta Nacional de Asistencia Médica Ambulatoria (National Ambulatory Medical Care Survey: NAMCS) para determinar si la prescripción de antibióticos en estos casos ha cambiado a lo largo del tiempo (entre 1989 y 1999) y para identificar los factores que permiten predecir la prescripción de antibióticos en general y de antibióticos no recomendados en particular.

El análisis se limitó a los adultos cuyo motivo de consulta fueron los "síntomas referidos a la garganta". Solo se incluyeron las consultas a los médicos de atención primaria: médicos de familia, médicos generales, internistas, médicos especialistas en adolescentes, geriatras y especialistas en medicina preventiva general. En los análisis, los especialistas en medicina de adolescentes se incluyeron junto con los médicos de familia, y los geriatras y los especialistas en medicina preventiva general con los internistas. Se excluyeron los menores de 18 años y los pacientes inmunodeprimidos con diabetes, infección por el virus de la inmunodeficiencia humana o cáncer. También se excluyeron los casos diagnosticados de sinusitis o con alguna otra causa de dolor de garganta que pudiera llevar al tratamiento con antibióticos. Asimismo, se excluyeron las consultas definidas como "no agudas". La muestra final consistió en 2244 consultas por dolor de garganta.

Se calcularon las tasas nacionales anuales de prescripción de antibióticos en pacientes con dolor de garganta y las tasas de tratamiento con antibióticos no recomendados. Solo se incluyeron los antibacterianos no tópicos. El tratamiento recomendado se definió como la administración de penicilina o eritromicina. Los datos fueron sometidos a un se- gundo análisis tras la exclusión de todos los diagnósticos distintos de la faringitis que pudieran justificar el tratamiento con antibióticos (bronquitis aguda, exacerbación aguda de bronquitis crónica, infección estafilocócica, infección bacteriana, infección gonocócica, linfadenitis, otitis media, otitis externa, neumonía, infecciones urinarias e infecciones cutáneas), lo cual redujo la muestra en un 17,5\% (1 852 consultas). Se calcularon los intervalos de confianza del 95\% (IC95\%) de los porcentajes estimados y se efectuó un análisis de regresión logística multivariado para identificar los factores que predecían la prescripción de antibióticos en general, y de antibióticos no recomendados en particular.

El número estimado de consultas anuales por dolor de garganta que cumplieron los criterios de inclusión fue de 6,7 millones (5,1 a 8,7 millones, según el año). Los diagnósticos más frecuentes fueron faringitis aguda $(41 \%)$, infección respiratoria de vías altas $(21 \%)$, amigdalitis aguda $(8 \%)$, infección estreptocócica $(6 \%)$, bronquitis aguda $(5 \%)$ y laringitis aguda (2\%). Se prescribieron antibióticos en el 73\% de las consultas (IC95\%: 70 a 76\%): antibióticos recomendados en el 23\% (IC95\%: 20 a 26\%) y no recomendados en el 49\% (IC95\%: 46 a 52\%). Entre los pacientes tratados con antibióticos, el 68\% (IC95\%: 64 a $72 \%$ ) recibió antibióticos no recomendados.

El uso de antibióticos recomendados disminuyó del 32\% en 1989 al 11\% en $1999(P<0,001)$. Hubo una disminución significativa del uso de la penicilina (del 17\% en 1989 al 6\% en 1999; $P<0,001$ ) y de la eritromicina (del 15\% en 1989 al 5\% en 1999; $P<0,001$ ). El uso de antibióticos no recomendados aumentó del $45 \%$ en 1989 al $56 \%$ en 1998, pero en 1999 disminuyó al 46\% $(P<0,001)$. Hubo un aumento significativo del uso de macrólidos de amplio espectro $(P<0,001)$ y fluoroquinolonas de amplio espectro $(P<0,001)$. La prescripción de antibióticos por los internistas disminuyó significativamente: del $74 \%$ en $1989-92$ al $64 \%$ en $1993-96$ y al $60 \%$ en $1997-99(P<0,01)$. No obstante, la prescripción de antibióticos no recomendados por estos especialistas aumentó de forma también significativa: del 66\% en 1989-92 al 73\% en 1993-96 y al 88\% en 1997-99 $(P=0,003)$. El uso de antibióticos no recomendados también aumentó entre los médicos de familia y los médicos generales, aunque de forma no significativa.

En el análisis multivariado, los factores relacionados de forma independiente con la prescripción de antibióticos fueron la edad (razón de posibilidades -OR-, 0,86 por cada decenio más; IC95\%: $0,79$ a 0,94$)$ y el hecho de que el médico fuera un generalista (OR, 1,54 en comparación con los médicos de familia; IC95\%: 1,10 a 2,14). Entre los pacientes tratados con antibióticos, los factores relacionados con la prescripción de antibióticos no recomendados 
fue el año civil (OR, 1,17 por año; IC95\%: 1,11 a 1,24). Al repetir el análisis tras la exclusión de todos los diagnósticos distintos de la faringitis que pudieran justificar el tratamiento con antibióticos, la proporción de consultas en las que se prescribieron antibióticos disminuyó del 73 al 71\% (IC95\%: 68 a 74\%) y la tendencia al mayor uso de fluoroquinolonas de amplio espectro dejó de ser significativa $(P=0,002)$.

Este estudio demuestra que la proporción de pacientes con dolor de garganta tratados con antibióticos en atención primaria (73\%) es muy superior a la prevalencia de los EBA (5 a 17\%) en adultos con este síntoma. El aumento del uso de macrólidos y fluoroquinolonas de amplio espectro es preocupante por dos motivos: su costo mucho más elevado en comparación con la penicilina y el riesgo mucho mayor de aparición de resistencia. Es necesario seguir tratando de alentar un uso apropiado de los antibióticos por parte tanto de los pacientes como de los médicos. (Linder JA, Stafford RS. Antibiotic treatment of adults with sore throat by community primary care physicians. A national survey, 1989-1999. JAMA 2001;268:1181-1186.)

\section{Los antidepresivos como factor de riesgo de cardiopatía isquémica}

La depresión es la cuarta causa más importante de discapacidad. Más del $45 \%$ de los pacientes hospitalizados tras un infarto de miocardio tienen depresión, diagnóstico psiquiátrico que constituye un factor de riesgo independiente de mortalidad y de morbilidad tras el infarto de miocardio. Dado que todavía no se ha esclarecido el papel de los antidepresivos en la cardiopatía isquémica, los autores de este estudio se propusieron determinar si estos fármacos constituyen un factor de riesgo, además de comparar el riesgo conferido por distintos fármacos y grupos de antidepresivos.

Para ello realizaron un estudio de casos y controles apareados por consulta, edad y sexo. Los pacientes fueron reclutados en nueve consultas de medicina general de una red de investigación (Trent Focus Collaborative Research Network). Se identificaron los casos nuevos de cardiopatía isquémica (angina de pecho, infarto de miocardio, cirugía coronaria) registrados entre el 1 de enero de 1995 y el 31 de diciembre de 1999. Como controles se seleccionaron personas que nunca habían recibido este diagnóstico.

Mediante un análisis de regresión logística múltiple condicional, se calcularon las razones de posibilidades (odds ratios: OR) y sus intervalos de confianza del 95\% (IC95\%). La principal variable de interés fue el uso de antidepresivos antes del diagnóstico de la cardiopatía isquémica. Se analiza- ron las interacciones entre los antidepresivos tricíclicos (ADT) y los inhibidores selectivos de la recaptación de serotonina (ISRS), entre la dosulepina (dotiepina) y la amitriptilina, y entre la dosulepina y la lofepramina. Los datos fueron ajustados en función de posibles efectos de confusión (diabetes, hipertensión, índice de masa corporal y consumo de tabaco) y se presentan las OR brutas y ajustadas. La relación entre la dosis y la respuesta fue analizada mediante una prueba de tendencia. Partiendo de un consumo de ADT del 10\% en los 5 años anteriores, se calculó que serían necesarios 804 conjuntos de casos y controles (cuatro controles por caso) para demostrar una OR de 1,5 con una potencia del 95\% y un nivel de significación del $5 \%$.

Entre los 74948 pacientes registrados en las nueve consultas hubo 933 casos nuevos de cardiopatía isquémica que cumplieron los criterios de inclusión. Los 516 hombres se emparejaron con 3081 controles del mismo sexo, y las 417 mujeres con 2435 controles del mismo sexo. Había datos sobre las prescripciones realizadas durante los últimos 7,5 años en los casos y 7,4 años en los controles.

La OR bruta de cardiopatía isquémica fue de 1,67 (IC95\%: 1,41 a 1,99) en los pacientes a los que alguna vez se les habían prescrito antidepresivos antes del diagnóstico de la cardiopatía isquémica, y este significativo aumento persistió tras el ajuste en función de los factores de confusión antes mencionados (OR, 1,63; IC95\%: 1,28 a 2,08). La prescripción previa de ISRS también se asoció con un aumento significativo de la OR bruta $(1,55$; IC95\%: 1,18 a 2,01), pero no de la OR ajustada (1,29; IC95\%: 0,89 a 1,87). En cambio, la prescripción previa de ADT se asoció con un aumento significativo tanto de la OR bruta $(1,67$; IC95\%: 1,38 a 2,01) como de la OR ajustada (1,56; IC95\%: 1,18 a 2,05). No hubo interacciones significativas entre el uso de ISRS y ADT.

Para determinar si estos resultados se debían a algún fármaco o grupo de fármacos, se repitieron los análisis para la amitriptilina, dosulepina y lofepramina (los tres ADT utilizados con mayor frecuencia). Las OR brutas estaban aumentadas con cada uno de ellos, pero las OR ajustadas solo siguieron estando significativamente aumentadas en el caso de la dosulepina (1,67; IC95: 1,17 a 2,36).

$\mathrm{El}$ análisis de la relación entre la dosis de dosulepina y la respuesta reveló que los pacientes tratados con dosis superiores a $50 \mathrm{mg}$ tenían mayor riesgo de cardiopatía isquémica que los tratados con dosis menores (OR bruta, 1,74; IC95\%: 1,26 a 2,41. OR ajustada, 1,72; IC95\%: 1,12 a 2,63). La prueba de tendencia fue altamente significativa $(P<0,0001)$.

Este estudio reveló que los pacientes con cardiopatía isquémica tenían mayores probabilidades que los controles de haber sido tratados con antide- 
presivos antes del diagnóstico. Después de ajustar los datos en función de los factores de confusión, la asociación se mantuvo con los ADT, pero no con los demás antidepresivos. No fue posible excluir la existencia de una asociación entre la cardiopatía isquémica y la depresión en sí misma, dado que el número de pacientes no tratados fue muy escaso. En el caso de la dosulepina, además de una asociación entre el consumo del fármaco y la cardiopatía isquémica, también se identificó una relación entre la dosis y la respuesta. La asociación es biológicamente verosímil, una vez que los ADT son antiarrítmicos de clase I y pueden causar hipotensión ortostática, efectos ambos que pueden precipitar un infarto de miocardio. Además, los ADT también aumentan la resistencia a la insulina, factor asociado con la aterogénesis. (Hippisley-Cox J, Pringle M, Hammersley V, Crown N, Wynn A, Meal A, et al. Antidepressants as risk factor for ischaemic heart disease: case-control study in primary care. BMJ 2001; 323:666-669.)

\section{Lugar de residencia e incidencia de cardiopatía coronaria}

Hoy en día, las enfermedades suelen explicarse por factores relacionados con el estilo de vida y la genética; al lugar de residencia no se le suele conceder gran importancia. No obstante, las características físicas y sociales del lugar de residencia pueden afectar tanto a la salud como a los comportamientos relacionados con ella, tales como el consumo de tabaco, la dieta y la actividad física.

En este estudio, los autores investigaron la relación entre las características del vecindario y la incidencia de cardiopatía coronaria en hombres y mujeres de cuatro regiones distintas de los Estados Unidos de América.

Como base se utilizaron los datos de un estudio prospectivo sobre la aterosclerosis, el Estudio del Riesgo de Aterosclerosis en las Comunidades (The Atherosclerosis Risk in Communities Study), realizado en cuatro comunidades de ese país: el Condado de Forsyth, Carolina del Norte; Jackson, Misisipi; los suburbios del noroeste de Minneapolis, Minnesota, y el Condado de Washington, Maryland. La cohorte constó de 15792 personas seleccionadas por muestreo probabilístico. Prácticamente todos los participantes del Condado de Washington y de los suburbios de Minneapolis eran blancos, al igual que el $85 \%$ de los participantes del Condado de Forsyth, mientras que todos los de Jackson eran negros. El examen inicial se realizó entre 1987 y 1989, cuando las personas tenían 45 a 64 años. Estas fueron contactadas telefónicamente una vez al año y sometidas a un examen médico cada 3 años.
Como indicador de las características socioeconómicas del vecindario se utilizó un índice compuesto cuyas variables fueron seleccionadas mediante un análisis factorial de los datos de los grupos de bloques censales (subdivisiones del censo de los Estados Unidos con una media de 1000 personas cada una). Se utilizaron seis variables que representaban tres dimensiones: riqueza e ingresos, nivel educacional y ocupación. Se calculó la puntuación $z$ de cada una de estas variables, cuya suma proporcionó la puntuación final del vecindario. Dichas puntuaciones finales oscilaron entre $-11,3$ y 14,4 (a mayor puntuación, mayor ventaja socioeconómica) y sobre esa base, las personas de cada raza fueron divididas en tres grupos más o menos del mismo tamaño. Más del $80 \%$ de los miembros de la cohorte seguían viviendo en el mismo vecindario 6 años después del inicio del estudio.

Los accidentes coronarios, identificados mediante los contactos telefónicos anuales, los exámenes médicos realizados cada 3 años y la inspección de las listas de altas de los hospitales locales y de los certificados de defunción, se definieron como un diagnóstico definitivo o probable de infarto de miocardio con hospitalización, la muerte por cardiopatía coronaria o un nuevo infarto de miocardio no reconocido (definido por la aparición, entre la primera consulta y las posteriores, de ondas Q mayores $u$ ondas $Q$ menores con alteraciones isquémicas del segmento ST-T o infarto). Las personas que determinaron si estos accidentes se habían producido desconocían la hipótesis investigada. En los análisis se incluyeron los accidentes ocurridos hasta el 21 de diciembre de 1997. La duración mediana del seguimiento fue de 9,1 años, y su duración máxima de 11,1 años. En la consulta inicial se registró información sobre los factores de riesgo cardiovasculares de cada participante.

Los valores basales de las características del vecindario y de los indicadores socioeconómicos personales se compararon mediante regresión lineal y regresión logística entre los individuos que presentaron cardiopatía coronaria y los que no la presentaron. Se calcularon las tasas de incidencia y, mediante regresión de Poisson, se ajustaron en función de la edad al inicio del estudio y de la región estudiada. Las razones de riesgo instantáneo de cardiopatía coronaria según los tres grupos de puntuaciones del barrio se calcularon mediante regresión de riesgos instantáneos proporcionales con ajustes en función de los indicadores socioeconómicos personales (ingresos, nivel educacional y ocupación) y de los factores de riesgo cardiovascular.

De los 15792 participantes examinados inicialmente, en el análisis se incluyeron 13 009, pertenecientes a 595 bloques censales (mediana de 16 participantes por bloque). De estas 13009 personas, 
615 sufrieron accidentes coronarios durante el período de seguimiento. Las tasas de incidencia de cardiopatía coronaria ajustadas por edades fueron del 7,3 por 1000 años-persona en hombres blancos, del 2,8 por 1000 en mujeres blancas, del 8,0 por 1000 en hombres negros y del 4,5 por 1000 en mujeres negras.

En general, la incidencia de cardiopatía coronaria disminuyó a medida que aumentaban las puntuaciones del vecindario. Aunque las asociaciones entre este índice y la incidencia disminuían tras el ajuste por los indicadores socioeconómicos personales, las diferencias entre los vecindarios menos favorecidos y los más favorecidos se mantuvieron. El hecho de residir en los vecindarios más desfavorecidos se asoció con un riesgo de coronariopatía 70 a $90 \%$ mayor en los blancos y 30 a $50 \%$ mayor en los negros, en comparación con la residencia en los vecindarios más favorecidos. La razón de riesgos de cardiopatía coronaria en las personas con menores ingresos residentes en los barrios más desfavorecidos, en comparación con las de altos ingresos residentes en los barrios más favorecidos, fue de 3,1 en los blancos (intervalo de confianza del 95\%: 2,1 a 4,8) y 2,5 en los negros (intervalo de confianza del 95\%: 1,4 a 4,5). Estas asociaciones no se modificaron tras el ajuste por los factores de riesgo de cardiopatía coronaria.

Estos resultados revelan que, incluso después de controlar los efectos de los indicadores socioeconómicos personales (ingresos, nivel educacional y ocupación) y de los factores de riesgo de cardiopatía coronaria, el hecho de vivir en vecindarios desfavorecidos se asocia con un aumento de la incidencia de cardiopatía coronaria. (Diez Roux AV, Merkin SS, Arnett D, Chambless L, Massing M, Nieto FJ, et al. Neighborhood of residence and incidence of coronary heart disease. N Engl J Med 2001;345:99-106.)

\section{Reducción del aborto en Bangladesh gracias a mejores servicios de planificación familiar}

En los países que han sufrido recientemente una transición demográfica se observa una relación inversa entre la tasa de fecundidad y las tasas de abortos y uso de anticonceptivos. A escala mundial, las tasas de fecundidad han disminuido de una media de seis niños por mujer en los años 50 a menos de tres hoy en día. Las tasas de uso de anticonceptivos han aumentado del 10\% en 1965 a más del 50\% en 1999. Cuando los anticonceptivos fracasan o no se usan, hay mujeres que recurren al aborto para evitar embarazos no deseados. Varios países en desarrollo tienen altas tasas de abortos ilegales, que conllevan un alto riesgo de morbilidad y mortalidad materna. En el mundo, aproximada- mente una de cada ocho muertes maternas se debe a abortos realizados sin garantías de seguridad. Aunque la existencia de mejores servicios de planificación familiar podría reducir el número de abortos y sus complicaciones, el desarrollo de los programas de planificación familiar también podría conducir al aumento de las tasas de abortos. No resulta fácil investigar el efecto de los servicios de planificación familiar sobre el aborto, no solo porque no hay datos fiables sino porque existen muchos factores de confusión. En Bangladesh se ha producido una transición demográfica de las más rápidas de todo el mundo. En los últimos 20 años, la tasa de fecundidad se ha reducido a la mitad, mientras que las tasas de aborto y uso de anticonceptivos se han triplicado, hasta llegar, respectivamente, al 55\% y 50 por 1000 actuales.

En este estudio se analizan los datos de dos zonas similares, típicas del Bangladesh rural, para investigar los efectos de la disposibilidad de mejores servicios de planificación familiar sobre las tasas de abortos.

La región rural de Matlab, Bangladesh, dispone desde 1966 de un Sistema de Vigilancia Demográfica que registra los datos sobre el desenlace del embarazo en las dos zonas estudiadas. Desde 1977, una de ellas (en adelante, zona PF) dispone de un Proyecto de Salud Maternoinfantil y Planificación Familiar que proporciona servicios de planificación familiar más accesibles y de mejor calidad que los proporcionados por el gobierno en el área de comparación (en adelante, zona C), donde las prácticas contraceptivas, la fecundidad, el aborto y la mortalidad materna son las características de las áreas rurales del país.

Los autores utilizaron los datos del Sistema de Vigilancia Demográfica para calcular la evolución de las tasas de aborto (número anual de abortos por 1000 mujeres de 15 a 49 años) entre 1979 y 1998 en ambas zonas. Además, analizaron los datos de las mujeres casadas de 15 a 49 años entrevistadas en las Encuestas de Matlab sobre Conocimientos, Actitudes y Prácticas relacionadas con la Anticoncepción, realizadas en 1984 y 1990, en las cuales se les preguntó, entre otras cosas, si deseaban tener más hijos, si estaban utilizando métodos anticonceptivos y, en caso afirmativo, cuáles. Estos datos permitieron calcular separadamente las tasas de embarazos deseados y no deseados y el porcentaje de embarazos de ambos tipos que concluían en abortos voluntarios.

A finales de los años setenta y principios de los ochenta, las tasas de abortos eran similares en ambas zonas, pero desde 1983 han aumentado en la zona $C$, mientras que en la zona PF han disminuido ligeramente. En consecuencia, a finales de los años noventa, en la zona PF había, por término medio, 5 
abortos menos por cada 1000 mujeres que en la zona C. En la actualidad, la tasa de abortos en la zona $C$ es más de tres veces mayor que en la zona $P F$. La diferencia entre las dos zonas fue significativa todos los años desde 1983 ( $P<0,001$ cada año).

En ambas áreas, tanto en 1984 como en 1990, casi la mitad de las mujeres no embarazadas que no usaban anticoncepción permanente dijeron que no querían tener más hijos. De las que sí querían, cerca de tres cuartos quedaron embarazadas en los 5 años siguientes. Las diferencias entre las dos zonas fueron mucho mayores en lo que se refiere a los embarazos no deseados. Así, cerca de la mitad de las mujeres de la zona $C$ que dijeron no desear más hijos tuvieron al menos uno en los 5 años siguientes; en el área PF, la tasa de embarazos no deseados fue significativamente menor. Esto indica que la disponibilidad de servicios de planificación familiar mejores y más accesibles en la zona PF les permitió evitar nuevos embarazos a las mujeres que no deseaban tener más hijos.

En ambas áreas y ambos períodos, la probabilidad de abortar fue mucho mayor en los embarazos no deseados que en los deseados. A su vez, la probabilidad de que los embarazos deseados acaba- ran en aborto fue considerablemente menor en la zona PF que en la zona $C$, diferencia que probablemente se deba al hecho de que a las mujeres de la zona PF quizás les resulte más fácil programar y espaciar sus embarazos.

En opinión de los autores, los resultados de este estudio revelan lo erróneas que pueden ser las conclusiones sobre la anticoncepción y el aborto que se pueden extraer de los análisis de los cambios a lo largo del tiempo, especialmente en situaciones de transición de la fecundidad. Si solo se hubiera considerado la zona $C$, se hubiera visto que mientras la fecundidad disminuyó en un tercio, el uso de anticonceptivos se triplicó y la tasa de abortos aumentó en un 30\%. Como estos cambios coincidieron con un aumento de las actividades de planificación familiar, se podría haber concluido que era esta la causa del aumento de la tasa de abortos.

Sin embargo, en la zona PF no se observó tal aumento de la tasa de aborto, a pesar de que también disponía de actividades de planificación familiar, pero más accesibles y de mejor calidad. En realidad fue esta mejor calidad y accesibilidad de las actividades de planificación familiar la que permitió mantener estable la tasa de aborto en la zona PF.
BASES

METODOLÓgICAS

PARA EVALUAR LA

VIABILIDAD Y EL

IMPACTO DE

PROYECTOS DE

TELEMEDICINA

(9)

\section{(3) (3)}

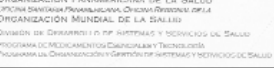

2001, $160 \mathrm{pp}$.

ISBN 9275323631

Código: OT 140

Precio: US\$24.00/

US\$18.00 en América Latina y el Caribe

\section{Bases metodológicas para evaluar la viabilidad y el impacto de proyectos de telemedicina}

El objetivo de esta publicación es sentar las bases metodológicas para evaluar la viabilidad y el impacto de la telemedicina. El enfoque no es sólo científico y técnico, sino que también abarca aspectos administrativos, legales, éticos, económicos, e incluso políticos. El énfasis recae en mejorar la calidad de la atención de la salud. A diferencia de lo que algunos creen, la telemedicina no reduce el personal especializado ni reduce la necesidad de equipamiento en los servicios que solicitan consultas, pero sí facilita los diagnósticos acertados y los tratamientos eficaces. Encierra ventajas para los pacientes, el personal de salud y la comunidad, pero sólo si los proyectos de telemedicina se planifican cuidadosamente y se evalúan sistemáticamente.

Creemos que esta publicación cubre un tema arduo con rigor y esperamos que su consulta mejore la calidad de la atención en los países de América Latina y el Caribe, ansiosos de entrar en una nueva faceta de la provisión de servicios de salud.

http://publications.paho.org • Fax: (301) 206-9789 • Correo electrónico:paho@pmds.com 